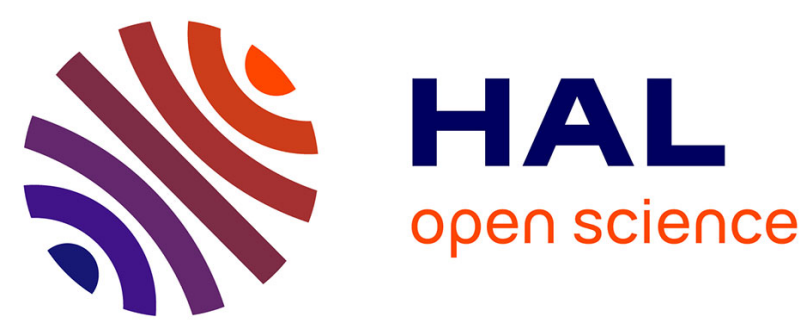

\title{
Synthesis of Hybrid Colloidal Nanoparticles for a Generic Approach to 3D Electrostatic Directed Assembly: Application to Anti-Counterfeiting
}

Romain Platel, Louis Vaure, Etienne Palleau, Simon Raffy, François Guerin, Delphine Lagarde, Robin Cours, Cécile Marcelot, Bénédicte Warot-Fonrose, Céline Nayral, et al.

\section{To cite this version:}

Romain Platel, Louis Vaure, Etienne Palleau, Simon Raffy, François Guerin, et al.. Synthesis of Hybrid Colloidal Nanoparticles for a Generic Approach to 3D Electrostatic Directed Assembly: Application to Anti-Counterfeiting. Journal of Colloid and Interface Science, 2021, 582, pp.1243-1250. 10.1016/j.jcis.2020.08.098 . hal-03373087

\section{HAL Id: hal-03373087 \\ https://hal.science/hal-03373087}

Submitted on 11 Oct 2021

HAL is a multi-disciplinary open access archive for the deposit and dissemination of scientific research documents, whether they are published or not. The documents may come from teaching and research institutions in France or abroad, or from public or private research centers.
L'archive ouverte pluridisciplinaire HAL, est destinée au dépôt et à la diffusion de documents scientifiques de niveau recherche, publiés ou non, émanant des établissements d'enseignement et de recherche français ou étrangers, des laboratoires publics ou privés. 


\section{Synthesis of Hybrid Colloidal Nanoparticles for a Generic Approach to 3D Electrostatic Directed Assembly: Application to Anti-Counterfeiting}

Romain Platel ${ }^{1}$, Louis Vaure ${ }^{1}$, Etienne Palleau ${ }^{1 *}$, Simon Raffy ${ }^{1}$, François Guerin ${ }^{1}$, Delphine Lagarde $^{1}$, Robin Cours ${ }^{2}$, Cécile Marcelot ${ }^{2}$, Bénédicte Warot-Fonrose ${ }^{2}$, Céline Nayral ${ }^{1}$, Fabien Delpech $^{1}$, Laurence Ressier ${ }^{1}$

1 - LPCNO, Université de Toulouse, CNRS, INSA, UPS, 135 avenue de Rangueil, 31077 Toulouse, France

2 - CEMES-CNRS, Université de Toulouse, 29 rue Jeanne Marvig, 31055 Toulouse, France

* Author to whom correspondence should be addressed: epalleau@insa-toulouse.fr / +33(0)5.61.55.96.72 


\begin{abstract}
Hypothesis

The capability of making 3D directed assembly of colloidal nanoparticles on surfaces, instead of 2D one, is of major interest to generate, tailor, and enhance their original functionalities. The nanoxerography technique, i.e. electrostatic trapping of nanoparticles on charged patterns, showed such 3D assembly potentialities but is presently restricted to polarizable nanoparticles with a diameter superior to $20 \mathrm{~nm}$. Hence, it should be possible to exploit a generic approach based on hybrid systems using larger nanoparticles as cargos to anchor smaller ones.
\end{abstract}

\title{
Experiments
}

A synthesis of hybrid nanoparticles in a raspberry-like configuration was performed using 50 $\mathrm{nm} \mathrm{SiO}_{2}$ nanoparticles and photoluminescent 3-5 nm InP@ $\mathrm{ZnS}$ (visible emission) or $\mathrm{PbS}$ (infrared emission) nanoparticles. Complete topographical and photoluminescent characterizations were carried out on hybrid nanoparticle patterns assembled by nanoxerography and systematically compared to patterns obtained from single photoluminescent nanoparticles.

\section{Findings}

The synthesis approach was generic. Every hybrid nanoparticle system has led to 3D assemblies with improved photoluminescent signals compared to $2 \mathrm{D}$ assemblies. Straightforward applications for anti-counterfeiting were illustrated. The versatility of the proposed concept is expected to be applied to other nanoparticles to make the most of their magnetic, catalytic, optical etc. properties in a wide range of applications, sensors and devices.

\section{Highlights}

* Versatile synthesis of hybrid colloidal nanoparticles dispersed in apolar solvents

* Nanoxerography of hybrid nanoparticles systematically lead to 3D patterns

* 3D hybrid assembly approach enhanced the nanoparticle original functionalities

Keywords: colloidal hybrid nanoparticle, nanoxerography, quantum dots, 3D directed assembly, electrostatic trapping, anti-counterfeiting, QR code 


\section{INTRODUCTION}

Owing to their unique physico-chemical properties, colloidal nanoparticles (NPs) are now part of the toolbox to fabricate active areas of promising nanodevices.[1-5] They have thus been widely employed in an extensive range of applications: sensors (temperature,[6] humidity,[7] gas,[8] chemical,[9] bioanalyses,[10,11] mechanics,[12]...), strain gauges,[13] memories,[14] SERS,[15] anti-counterfeiting applications,[16] etc.

One prerequisite to benefit from the tuned properties of NPs in concrete realizations is to integrate them and direct their assembly onto specific areas of rigid or flexible surfaces. Thus, numerous directed assembly strategies are used and developed to finely control their localization and structuration onto surfaces.[17,18] The capability of these integration approaches to make 3D assemblies/nanostructures instead of 2D ones is of major interest to generate, tailor, and enhance the NP original functionalities.[19]

Among the available directed assembly methods, nanoxerography, i.e. electrostatic assembly induced by charged patterns written onto electret surfaces, is a powerful technique to address a large variety of colloidal nanoparticles.[20] Its capability to realize 3D assembly was demonstrated with photoluminescent NPs which enabled a tuning of the photoluminescent signal that depends on the assembly thickness.[21] These technique potentialities were successfully applied to fabricate anti-counterfeiting markers considering multi security levels,[21] the biocompatible aspect,[22,23] the reading equipment (smartphone [24] or a dedicated optical setup [21]) and their industrial scaling up fabrication.

\section{$[22,24,25]$}

Unfortunately, 3D assembly by nanoxerography is limited to polarizable NPs with "large" diameter above 20-25 $\mathrm{nm},[26]$ which represents a severe restriction. Indeed, in the case of quantum dots (QDs) for instance, the lack (or the weakness) of quantum confinement effect in NPs larger than $10 \mathrm{~nm}$ makes them less desirable, their synthesis with narrow size 
distribution being in addition often very challenging like for example in the case of the strategic InP QDs.[27]

In the present study, we propose a flexible hybrid approach to enlarge the range of applicable NPs for 3D assembly by nanoxerography. A synthesis of hybrid raspberry-like NPs is evaluated where large 3D-assemblable $\mathrm{SiO}_{2} \mathrm{NPs}$ are used as vector to carry nanometric particles of interest. This generic protocol is tested with 3-4 nm InP@ZnS QDs and $5 \mathrm{~nm} \mathrm{PbS}$ QDs and applied to realize respectively cadmium-free and infrared-readable anticounterfeiting photoluminescent markers.

\section{EXPERIMENTAL}

\subsection{Materials}

Indium(III) chloride (99.999\%), zinc stearate (99\%), tris(diethylamino)phosphine (97\%), trioctylphosphine (>99\%), elemental sulfur ( $\geq 99 \%)$, oleylamine $(90 \%)$, octadecene technical grade (90\%), oleic acid technical grade (90\%), toluene ACS reagent $(\geq 99.5 \%)$, pentane ACS reagent $(\geq 99.5 \%)$, ethanol ACS reagent $(\geq 99.5 \%)$, hexane ACS reagent $(\geq 99.5 \%)$, methyl isobutyl ketone reagent, ammonia solution (28.0-30.0\%), dodecyltriethoxysilane, (3mercaptopropyl)triethoxysilane ( $\geq 80 \%)$, polymethylmethacrylate (PMMA, 996K) are all purchased from Sigma Aldrich. $\mathrm{PbCl}_{2}$, ultra dry (99.999\%) and tetraethoxysilane $(+99,999 \%)$ are purchased from Alfa Aesar. Methanol $\geq 99.7 \%$ is purchased from Honeywell. All chemicals are used as received and without any further purification.

3-4 nm InP@ZnS (quantum yield around 50\%) and $5 \mathrm{~nm} \mathrm{PbS} \mathrm{(quantum} \mathrm{yield} \mathrm{around} \mathrm{40 \% )}$ QDs dispersed in hexane were produced following established protocols published respectively by Tessier et al. [28] and Moreels et al. [29] After synthesis, the surface ligands on PbS QDs are oleic acid with possible traces of oleylamine and the ones on InP@ZnS QDs are trioctylphosphine (TOP). 


\subsection{Synthesis of photoluminescent hybrid nanoparticles}

All manipulations were performed under an argon atmosphere using Schlenk line techniques, or in an argon-filled glovebox.

\section{Synthesis and functionalization of $\mathrm{SiO}_{2}$ cargo nanoparticles}

In a typical experiment, tetraethoxysilane $(2.00 \mathrm{~mL}, 8.9 \mathrm{mmol})$ is added $(1 \mathrm{drop} / \mathrm{s})$ to a solution of ammonia $(4.72 \mathrm{~mL})$ in methanol $(20 \mathrm{~mL})$ in a Schlenk tube at $35^{\circ} \mathrm{C}$ under stirring (600 rpm). The solution is let under stirring for 12 hours which leads to the synthesis of $\mathrm{SiO}_{2}$ nanoparticles.

To further use them as cargo nanoparticles, the surface of the as-synthesized $\mathrm{SiO}_{2} \mathrm{NPs}$ is functionalized with a mixture of dodecyltriethoxysilane (DDS) and mercaptopropyltriethoxysilane (MPS). The mixture of these two silanes is made prior to injection. The amounts of the silanes are comprised between 1 and 10 molecules per $\mathrm{nm}^{2}$ of silica NPs for MPS and 10 and 100 molecules per $\mathrm{nm}^{2}$ of silica NPs for DDS. The calculated amount of both MPS and DDS silanes is based on the average size of the silica cargo nanoparticles, measured by analysis of TEM images. Supposing nanoparticles are spherical, the surface area of a single one is thus estimated. The solution is stirred at the same

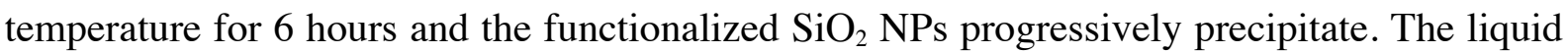
phase is discarded, and the solid is solubilized in $20 \mathrm{~mL}$ of hexane under ultrasounds. The obtained NPs are stable for weeks without further treatment. 


\section{Synthesis of hybrid $\mathrm{SiO}_{2}$-QDs particles}

In a typical experiment, a solution of functionalized $\mathrm{SiO}_{2}$ nanoparticles (1 MPS and 10 DDS molecules $/ \mathrm{nm}^{2}$ ) with the concentration of $1.1110^{13} \mathrm{NPs} / \mathrm{mL}$ is prepared in $9 \mathrm{~mL}$ of hexane.

A solution of $1 \mathrm{~mL}$ of QDs (either InP@ZnS or PbS ones) with the concentration of $1.0010^{16}$ QDs/mL is added and left for stirring for 1 hour at room temperature. MPS ligands on $\mathrm{SiO}_{2}$ nanoparticles have a silane moiety which will link to the silica nanoparticle surface, leaving free their thiol moiety. Once mixed with the QD solution of interest, this thiol moiety will attach to a metal atom at the surface of QDs $(\mathrm{Pb}$ or $\mathrm{Zn})$, replacing the native ligands, and creating covalent bonds between QDs and silica nanoparticles.

The hybrid $\mathrm{SiO}_{2}$ - QDs particles are finally isolated by centrifugation (15000 rpm for $15 \mathrm{~min}$ ) and suspended in hexane.

Washing steps conduced after synthesis of hybrid nanoparticles should prevent the final solution from the presence of free QDs. However, a few single QDs may still be free in the solution after washing, or even be desorbed of the surface of the silica nanoparticles. This amount of free QDs has not been quantified, but it can be seen on some TEM images.

It is worth noting that the concentration of both MPS and DDS silanes has been optimized experimentally, but no further control of their density has been made afterwards. Even if there is no direct proof that final densities of silanes at the surface of nanoparticles corresponds to the ones introduced/expected, the fact that hybrid nanoparticles are stable in apolar solvents and luminescent demonstrate that both respective functionalizations were successful.

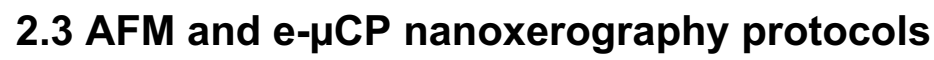

Nanoxerography protocols conducted in this study were based on previously published ones.[24,30] 
In a typical experiment, charged patterns of specific designs are first created on the surfaces of samples composed of $200 \mathrm{~nm}$ thick PolyMethylMethAcrylate (PMMA) thin films spincoated on $10^{16} \mathrm{P}$-doped Si wafer substrates. Si substrates act as counter electrodes and PMMA is used as electret for its excellent charge storage properties and ease of implementation by simple spin coating.[31] Charge writing is performed either via a polarized atomic force microscope (AFM) tip $[23,30]$ or by electrical microcontact printing $(\mathrm{e}-\mu \mathrm{CP}) \cdot[22,24] \mathrm{E}-\mu \mathrm{CP}$ and AFM are two charge writing techniques used in nanoxerography processes that complement each other depending if scaling up / charged patterns larger than $100 \mu \mathrm{m}$ are required, if there is a need for more flexibility/complexity or if characterizations of charged patterns by Kelvin Force Microscopy are mandatory.

Dielectrophoretic forces are induced by electric field gradients which can be created in a similar manner by positively or negatively charged patterns.[32] Consequently, in this study, only positively charged patterns were considered since their charge decay is slower than for the negatively charged ones.

In the AFM configuration case, voltage pulses $(1 \mathrm{~ms}, 50 \mathrm{~Hz}$, up to $80 \mathrm{~V}$ amplitude $)$ are applied by an external voltage generator in between a conductive AFM tip and the Si substrate. Experiments are run on an ICON microscope from Brüker using (42 N/m, $285 \mathrm{kHz})$ from NanoWorld.

In the e- $\mu \mathrm{CP}$ configuration case, a conductive stamp, made of cyclic olefin copolymer (COC) covered with a $50 \mathrm{~nm}$ gold layer, is brought in contact with the PMMA surface using a desktop nanoimprint tool CNI from NILT. Continuous voltages (typically $+45 \mathrm{~V}$ with a compliance of $100 \mathrm{~mA}$ ) are applied for $45 \mathrm{~s}$.

Charged samples were then immersed into colloidal dispersions of interest for 30-45 seconds, removed and naturally dried in air. 


\subsection{Characterizations of synthesized nanoparticles or nanoparticle assemblies}

\subsubsection{Characterizations of nanoparticles in solution}

\section{TEM}

Samples for typical TEM analyses were prepared in the glovebox by dropping a single drop of a dilute solution of washed particles in toluene or pentane onto a carbon-covered copper grid and allowing the solvent to dry slowly. Low and high magnification TEM analysis were performed on a JEOL JEM 1400 electron microscope operating at $120 \mathrm{kV}$ or a TECNAI F20 electron microscope operating at $200 \mathrm{kV}$. The nanoparticles were not sensitive to the electron beam and no degradation of the assembly was observed. The typical size distributions were determined by measuring at least 250 particles with Fiji (imageJ) software.[33]

\section{Photoluminescence}

Visible photoluminescence (PL) spectra were acquired with a PTI Fluorescence Master System equipped with a xenon lamp $\left(\lambda_{\text {excitation }}=450 \mathrm{~nm}\right)$, a monochromator and a UV-VIS Hamamatsu photomultiplier. Short-wave infrared or SWIR photoluminescence spectra were obtained using cw TiSa laser excitation at $800 \mathrm{~nm}$ and recorded by an InGaAs photodiode array after dispersion through a Princeton Instruments spectrometer. Quartz cuvettes $(10 \mathrm{~mm}$ pathlength) were initially cleaned with aqua regia followed by washing with toluene and ethanol. The cuvettes were dried in an oven $\left(120^{\circ} \mathrm{C}\right)$ to remove any traces of solvent. All tested NPs had an optical density between $0.05-0.17$ at the excitation wavelength $(450 \mathrm{~nm})$. UV-Visible and SWIR absorption spectra were measured with a Cary Series UV-Vis-SWIR spectrometer using cells of $2 \mathrm{~mm}$. 


\subsubsection{Characterizations of nanoparticle assemblies on surfaces}

\section{AFM}

The topography of nanoparticle patterns was analyzed in regular tapping mode at $0.5 \mathrm{~Hz}$ using an ICON atomic force microscope from Brüker equipped with ARROW NCR tips (42 N/m, $285 \mathrm{kHz}$ ) from NanoWorld.

\section{Optical microscopy}

Optical images were taken using a conventional direct optical microscope from Olympus in bright field mode. Samples were illuminated with a classical white source.

\section{SEM}

Samples for typical SEM analyses were prepared by covering their surfaces of the nanoparticle assemblies with a nanometric layer of gold using a SC7610 Sputter Coater. This preparation allows decreasing the charge effect due to the accumulation of electrons on the surface of the samples, which is relatively strong in our case due to the presence of the PMMA electret surface. However, the samples stayed quite sensitive to the electron beam thus leading to some observable surface holes and cracks on 3D assemblies. SEM analyses were performed on a FEG-SEM JEOL 7800F operating at $5.0 \mathrm{kV}$. The samples were tilted by $40^{\circ}$ to facilitate the visualization of the $3 \mathrm{D}$ aspect of NP assemblies.

\section{Photoluminescence}

Visible photoluminescence images were obtained with an inverted microscope IX73 (Olympus) under reflective light configuration. The light source is a pE-300 White (CoolLed) coupled with a filter cube (41012 from Chroma) and a camera (Grasshopper3 USB3 GS3-U323S6C-C from Flir).

SWIR photoluminescence images of nanoparticle assemblies were obtained after excitation by a $980 \mathrm{~nm} \mathrm{cw}$-laser diode. The excitation spot on the surface was estimated to be $200 \mu \mathrm{m}$ in 
diameter. SWIR PL images were then recorded by a SWIR camera (PHF-SNAKE-640 type) from PhotonFocus. A spectral bandpass filter (1200-1350 nm) was used to remove any scattered laser light on the camera. The sample was mounted on a motorized microscope stage from Märzhäuser to allow sample scanning below the laser spot. The various obtained images were used to reconstruct the whole PL image of the assemblies.

\section{RESULTS AND DISCUSSION}

\subsection{Hybrid nanoparticles: a strategy for indirect 3D directed assembly of small nanoparticles on surfaces by nanoxerography}

Previous results on nanoxerography demonstrated that some specifications are required to access selective electrostatic 3D assembly of colloidal nanoparticles through this technique [26]:

- The quantity of free ligands should be minimized in the colloidal dispersion to prevent unwanted screening of charged patterns.

- The concentration of NPs should be maximized to reach higher assembly thicknesses.[21]

- NPs of interest have to be uncharged in order to avoid electrostatic repulsion between them during the 3D construction of the assembly.

- NPs of interest have to be polarizable to be sensitive to the dielectrophoretic forces generated by the charged patterns.[34] To attract polarizable NPs on any type of charged patterns (positively or negatively), they need to be more polarizable than their dispersing solvent, which is verified when the used solvent has a low dielectric coefficient like hexane or toluene.

- The size of the NPs should be maximized in order to enhance the dielectrophoretic forces. These forces indeed increase as a function of the NP radius to the cube.[32] So far, 
examples of successful 3D directed assemblies by nanoxerography had been realized with NPs with a diameter superior to $20 \mathrm{~nm}$. [21]

This last point is a real bottleneck since it restricts the capability of nanoxerography to allow the 3D directed assembly of any type of nanoparticles, especially quantum dots whose typical diameters of several nanometers are too small. In those cases, the involved dielectrophoretic forces only lead to 2D mono/bilayered assemblies [34] and are not high enough to attract the deposit of extra layers of NPs.

To circumvent this limitation, the proposed approach is to create hybrid raspberry-like nanoparticles (HNPs). The combination of various components on the same nano-object is a relevant strategy to access or tailor new properties and further integrate nanoparticles into functional applications.[35,36] Here, the idea is finding a neutral but polarizable NP, large enough to lead to 3D assembly by nanoxerography, and large enough to anchor with adequate functionalization smaller NPs on its surface.

To explore this path, an ideal NP candidate for vector has been selected: $50 \mathrm{~nm} \mathrm{SiO}_{2} \mathrm{NPs}$ dispersed in hexane. It can be used as a cargo particle which can graft smaller NPs of interest (here demonstrated with QDs) on its surface (Figure 1a). In that way, QDs could be incorporated in 3D assemblies. Consequently, the global photoluminescent signal coming from their assembly in 3D in a hybrid configuration is expected to be higher compared to $2 \mathrm{D}$ assemblies obtained with single free QDs. It is interesting to note that in the case of another directed assembly technique, the so-called convective self-assembly one, a similar strategy has been recently employed to topographically trap in microstructures, large enough polystyrene NPs anchored with smaller photoluminescent NPs.[37] 
The 3D assemblies of HNPs were tested following the nanoxerography processes detailed in the Experimental Section and illustrated in Figure 1b.

\subsection{Characterizations of hybrid $\mathrm{SiO}_{2}-\mathrm{InP} @ Z \mathrm{ZnS}$ HNPs}

The hybrid approach proposed here for 3D assembly is first illustrated by the synthesis of HNPs combining $\mathrm{SiO}_{2}$ cargo NPs with InP@ZnS QDs. The main challenge of this approach is the combination of the apolar QDs and the polar $\mathrm{SiO}_{2}$ NPs prepared in methanol. The transfer of these latter in hexane (suitable for nanoxerography) is not trivial and requires a fine adjustment of specific parameters: temperature of reaction, amounts of ligand for exchange, washing steps for transfer. The various steps of the synthesis and details are described in the Experimental Section 2.2.

Figure 2 shows the absorption and emission spectra as well as the TEM images of $\mathrm{SiO}_{2}$ cargo NPs, InP@ZnS QDs and their combination in $\mathrm{SiO}_{2}$-InP@ZnS HNPs. As can be seen in Figure 2b, the InP@ZnS QDs are homogeneously distributed over the surface of the $\mathrm{SiO}_{2}$ NPs. Most importantly, the size and the optical properties of the QDs are fully preserved when combining the two types of NPs.

\subsection{Photoluminescence comparison of 2D and 3D nanoparticle patterns assembled by nanoxerography}

AFM nanoxerography experiments were realized on samples featuring series of 3 square patterns charged via different increasing applied voltages from left to right ranging from +45 $\mathrm{V}$ to $+80 \mathrm{~V}$ (Figure 3a).

Assemblies were performed with the three types of NP dispersions described in Figure 2: $\mathrm{SiO}_{2}$ NPs, InP@ZnS QDs and $\mathrm{SiO}_{2}$ - InP@ZnS HNPs. 
Typical assembly results were characterized by AFM in tapping mode, bright field optical microscopy and PL measurements. They confirm that naked $\mathrm{SiO}_{2} \mathrm{NPs}$ are well assembled in $3 \mathrm{D}$ and the higher the surface potential of the charge pattern the thicker the $3 \mathrm{D}$ assembly (Figure 3a). Assembly thicknesses of more than $1.3 \mu \mathrm{m}$ high (more than 31 layers) were obtained in the most highly charged conditions (+80 V applied to the AFM tip).

In the case of single InP@ZnS QDs, nanoxerography experiments lead to 2D mono/bilayered assemblies (Figure 3b). Increasing the surface potential of the charged patterns increases the density, first of monolayered QDs assemblies for the less charged patterns and second of bilayered assemblies for the most charged patterns (Figure S1a of Supporting Info). The PL signal increases accordingly but remains quite difficult to detect overall. A clear PL signal only appeared for the densiest bilayered assemblies.

Assembly results for $\mathrm{SiO}_{2}$-InP@ZnS HNPs perfectly combine the two previous features and lead to 3D luminescent assemblies (Figure 3c). The thicker the 3D assembly, the higher the photoluminescence. The graph of Figure 3d illustrates the linear evolution of the normalized photoluminescence (in arbitrary units) of 3D assembly of HNPs made by nanoxerography as function of their thicknesses. Obtained thicknesses can reach up to 31 layers of HNPs, comparable to thicknesses obtained in the case of naked $\mathrm{SiO}_{2}$ NPs. The maximum photoluminescence signal emitted by the densiest bilayered assembly only composed of InP@ZnS QDs (Figure 3b) is also reported on the graph with a red diamond. The value of this photoluminescence signal is equivalent to typical one obtained with a $150 \mathrm{~nm}$ thick $3 \mathrm{D}$ HNP assemblies. The results indicate that the HNPs approach lead to 3D assembly, 8 times more luminescent (black dash lines for the $1.3 \mu \mathrm{m}$ thick HNP assembly) than 2D bilayered assembly of single InP@ZnS QDs. It is in good agreement with the number of InP@ZnS emitters that is involved in both 2D and 3D assemblies (cf. calculations in SI). By considering 
an ideal total covering of $\mathrm{SiO}_{2}$ NPs by InP@ZnS QDs, the photoluminescent gain could possibly reach 32 (cf. calculations in $\mathbf{S I}$ ).

Figure 4a depicts the realization of $159 \mu \mathrm{m}$ wide $\mathrm{QR}$ codes obtained by e- $\mu \mathrm{CP}$ nanoxerography respectively composed of InP@ZnS NPs and $\mathrm{SiO}_{2}-\mathrm{InP} @ \mathrm{ZnS}$ HNPs. These micrometric QR codes are only visible when excited with a blue LED excitation at $460 \mathrm{~nm}$ and have an orange-red emission at $631 \mathrm{~nm}$. They contain the coding of 185 alphanumeric characters which is referring to an example of secured traceability data that can be tagged on a product. This example clearly demonstrates the improvement in the photoluminescence signal due to $3 \mathrm{D}$ assembly. Thus, only the captured PL image in the $3 \mathrm{D} / \mathrm{HNP}$ case is contrasted enough to be directly read by a reader application (Figure S2 of SI). SEM characterizations (Figure 4b) of the assembly confirm the 3D aspect of the QR code. It also reveals that some HNPs are non-selectively deposited outside the QR code. However, their specific PL signal neither really affects the global QR code signal nor its contrast. Furthermore, TEM characterizations (Figure 4c) on a cross-section of the 3D assembly obtained by focused ion beam (Figure S3 of SI) prove that the process of nanoxerography doesn't disturb the grafting of the small InP@ZnS NPs on the $\mathrm{SiO}_{2}$ ones once assembled.

The generic aspect of the HNP approach proposed here has been investigated by grafting another type of QDs on $\mathrm{SiO}_{2}$ cargo NPs: $5 \mathrm{~nm}$ PbS QDs. Their synthesis has been described in the Experimental Section 2.2. Figure 5a illustrates typical absorption/emission spectra of $\mathrm{SiO}_{2}-\mathrm{PbS}$ HNPs. As for single InP@ZnS QDs, it was verified that nanoxerography of single $\mathrm{PbS}$ QDs leads to 2D monolayered or bilayered assembly (Figure S1b of SI). However, no 3D assembly could be obtained. In contrast, 3D assembly of $\mathrm{SiO}_{2}-\mathrm{PbS}$ HNPs could be achieved.

Results on both hybrid nanoparticle systems confirm that the thickness of the 3D assembly increases linearly with the surface potential of the charge pattern and so thickest 
assemblies are obtained for the mostly charged patterns (Figure S4a of SI). Furthermore, a short optimized immersion time in the 5-45 seconds range into the corresponding colloidal solution to access thickest assemblies (Figure S4b of SI) is in good agreement with previously reported assembly kinetics.[26] It proves that 3D assembly by nanoxerography of such hybrid systems is mainly directed by dielectrophoretic forces generated by the charge patterns. Hybrid nanoparticles keep piling up in a 3D manner as long as they feel dielectrophoretic forces larger than diffusion ones. For longer immersion time, the diffusion phenomenon overcomes dielectrophoretic attraction and 3D nanoparticle assemblies start breaking up little by little, down to single monolayers. The maximum assembly thickness led by the described 3D mechanism thus seems limited by the maximum initial amplitude of the dielectrophoretic forces (i.e. the surface potential of the charge patterns) reached in our experiment conditions.

An example of 2D assembly of single PbS NPs and of 3D assembly of $\mathrm{SiO}_{2}-\mathrm{PbS}$ HNPs by e- $\mu \mathrm{CP}$ nanoxerography representing the logo of the LPCNO lab is captured by a dedicated optical setup (Figure 5b). This logo has an infrared emission around $1.3 \mu \mathrm{m}$ when excited with a laser at $980 \mathrm{~nm}$. Further SEM and TEM characterizations on a cross-section of such 3D assembly obtained by focused ion beam (Figure S5 of SI) depicted identical conclusions than for 3D assembly of $\mathrm{SiO}_{2}$-InP@ZnS HNPs. The comparison of two LPCNO logos by IR imaging proves the enhancement of signal and so contrast in the 3D assembly case (Figure 4b). We thus confirm the relevance of 3D assembly obtained by the HNP approach and validate its capability to be extended to other types of small NPs/QDs.

\subsection{Application perspectives considering industrial constraints}

Fabricating anti-counterfeiting markers with photoluminescent nanoparticles is an elegant approach, especially because of the ability of finely tuning the nanoparticle optical properties.[16,38] It thus can address various levels of security both on a reading and a 
fabrication point of view. Moreover, the use of QR codes as markers present multiple well known advantages: free license technology, easy implementation and reading, good amount of coded data and the capability to undergo partial physical damages owing to information redundancy. To meet industrial requirements in terms of mass production, speed, cost, targeted products, large scale printing methods have been developed, the most widespread being inkjet printing. [16,38] However, the formulation of colloidal inks remains a challenging task (optimizing solvent compatibility with cartridge, viscosity, concentration, stabilization) which directly impacts the printing resolution and induces possible clogging.

Nanoxerography can compete with inkjet printing if the charge injection step is performed with parallel in-line compatible processes like electrical microcontact printing $[22,24]$ or electrical nanoimprinting.[25] Furthermore, to tag different types of surfaces, it is mandatory to get rid of the silicon substrate, only useful as counter electrode for the injection step in nanoxerography. That is why, a transfer protocol based on a sacrificial water-soluble polymer layer was previously developed.[25] This concept was successfully applied to incorporate CdSe@CdS nanoparticle based anti-counterfeiting markers into official documents.[24]

Now, with the capability of producing by nanoxerography versatile 3D anticounterfeiting markers with improved photoluminescent performances like the ones demonstrated in the present work, an enlarged panel of concrete applications including Restriction of Hazardous Substances (RoHS) certified or IR responsive ones may be targeted. 


\section{CONCLUSIONS}

This study extends the range of NPs that can be incorporated in 3D assemblies by the nanoxerography process, previously restricted to NPs with diameter larger than $20 \mathrm{~nm}$. Such limitation was tackled by introducing a hybrid NP synthesis approach where a large $50 \mathrm{~nm}$ $\mathrm{SiO}_{2} \mathrm{NP}$ is used as a cargo to anchor smaller (several $\mathrm{nm}$ ) NPs on its surface. 3D assembly of hybrid $\mathrm{SiO}_{2}$-InP@ $\mathrm{ZnS}$ and $\mathrm{SiO}_{2}$-PbS NPs were successfully demonstrated. The obtained 3D assemblies were applied to the fabrication of anti-counterfeiting markers which emit and so are only visible at specific visible or IR wavelengths (depending on the used NPs) when excited at specific wavelengths. Having 3D NP assembly instead of a 2D one allows among other things the enhancement or the rising of new properties. Here, the photoluminescence signal of the 3D assembly linearly increased as a function of the assembly thickness and a maximum gain by 8 -fold was achieved.

We thus provide the proof of concept of the 3D assembly through nanoxerography of HNPs. The use of more complex heterostructure including more functions/properties can be envisioned: for example, the $\mathrm{SiO}_{2}$ cargo particles used in this study don't take action in the photoluminescence signal emitted by the assembly; so it would be possible to tune or enhance even more the signal by using an "active" cargo that could induce PL-plasmonic interactions.[39]

We expect the versatility of the proposed concept to be applied to other types of NPs to make the most of their magnetic, catalytic, optical, etc. properties in a wide range of applications, sensors and devices. 
(a)

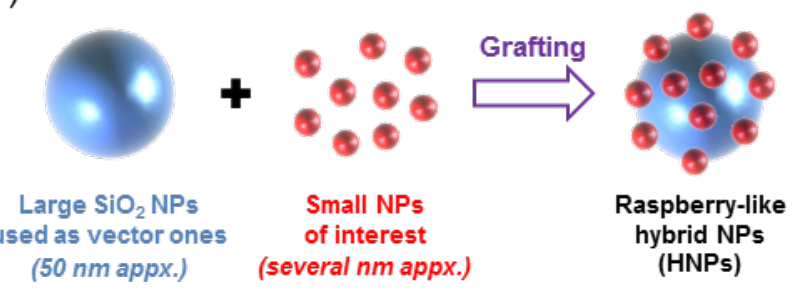

(b)

1/ Charge writing

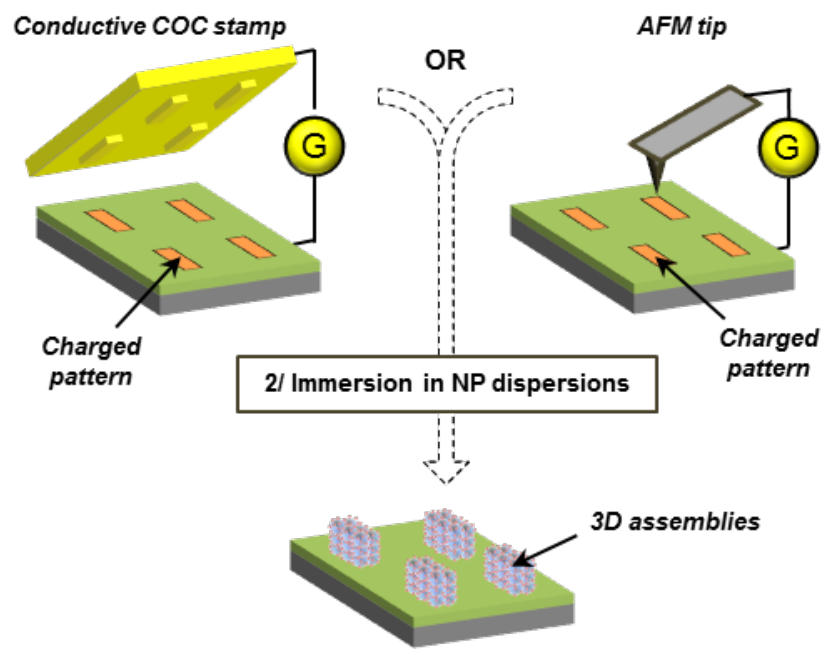

Figure 1. (a) Synthesis of raspberry-like hybrid NPs by grafting small NPs/QDs of interest onto larger $50 \mathrm{~nm} \mathrm{SiO} \mathrm{S}_{2} \mathrm{NPs}$. (b) Principle of the electrical microcontact printing (e- $\mu \mathrm{CP})$ or atomic force microscopy (AFM) nanoxerography applied to these hybrid NPs to fabricate directed $3 \mathrm{D}$ patterns. 


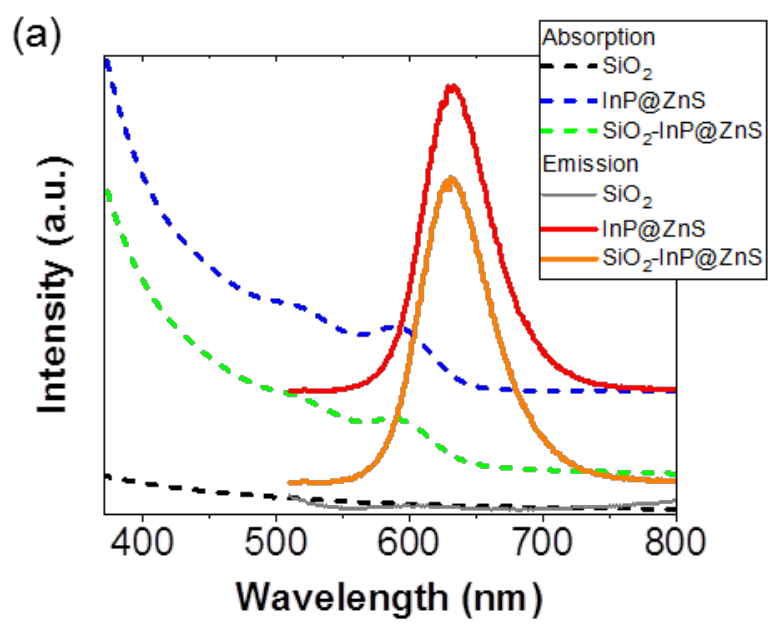

(b)
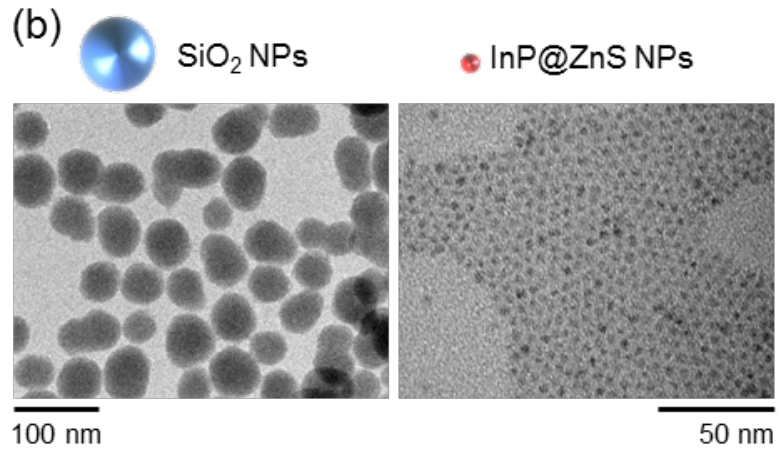

$\mathrm{SiO}_{2}-\mathrm{InP} @ \mathrm{ZnSHNPs}$

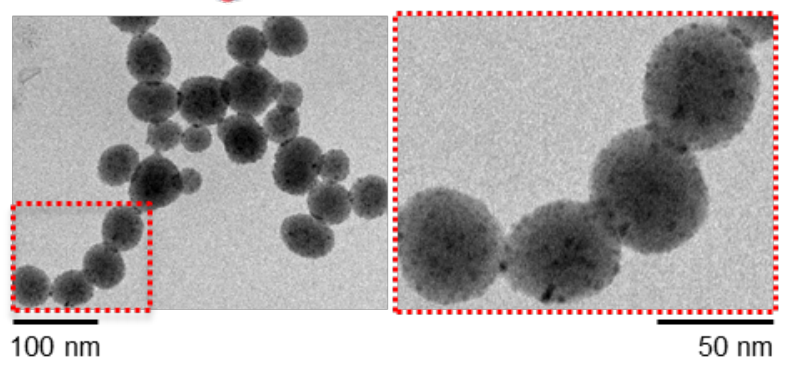

Figure 2. (a) Emission and absorption spectra of $\mathrm{SiO}_{2}$, InP@ZnS and $\mathrm{SiO}_{2}$-InP@ZnS hybrid NPs. (b) Transmission electron microscopy (TEM) images of the corresponding NPs. 
(a) $\mathrm{SiO}_{2} \mathrm{NPS}$

(b) • InP@ZnS NPs
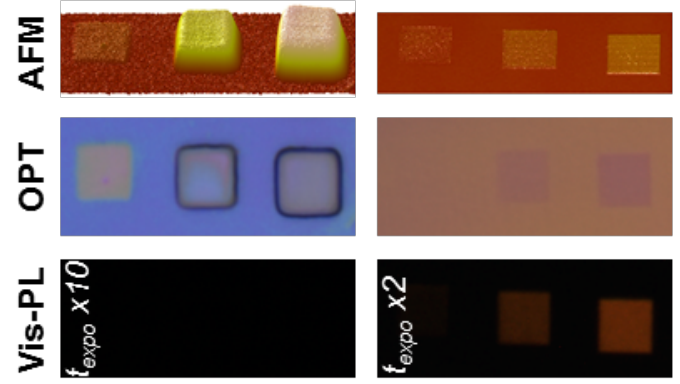

(c)
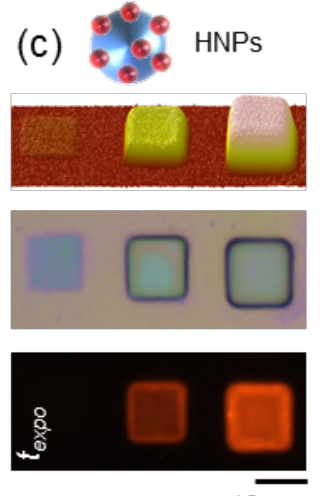

$10 \mu \mathrm{m}$ (d)

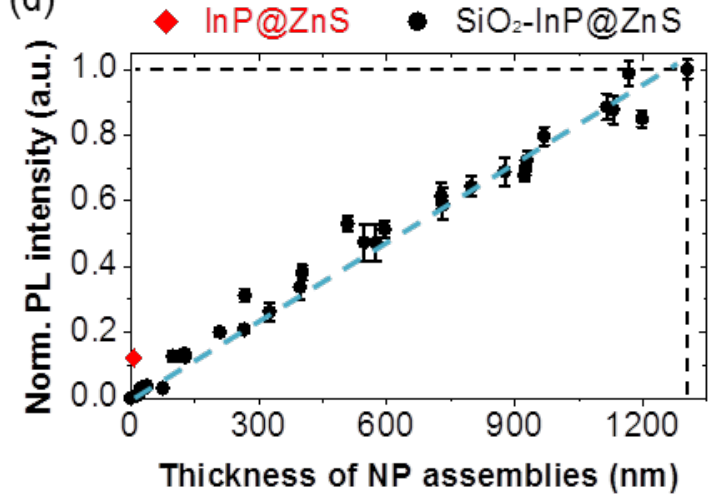

Figure 3. (a-b-c) Microscopy characterizations of $\mathrm{SiO}_{2}$, InP@ZnS and $\mathrm{SiO}_{2}-\ln \mathrm{PP} \mathrm{ZnS} N \mathrm{NP}$ assemblies: 3D AFM topographical images, bright field optical microscopy images and photoluminescent mappings (at $631 \mathrm{~nm})$ under a blue $(460 \mathrm{~nm})$ LED irradiation. Vis-PL images are taken at different exposure times $\left(t_{\text {expo }}\right)$. (d) Correlation between the thickness of $\mathrm{SiO}_{2}-\mathrm{InP} @ \mathrm{ZnS} \mathrm{NP}$ assemblies, using images displayed in (c), and their PL intensity (in red levels); the maximum of red PL intensity obtained from 2D InP@ZnS assemblies is shown for comparison (diamond label). 
(a)
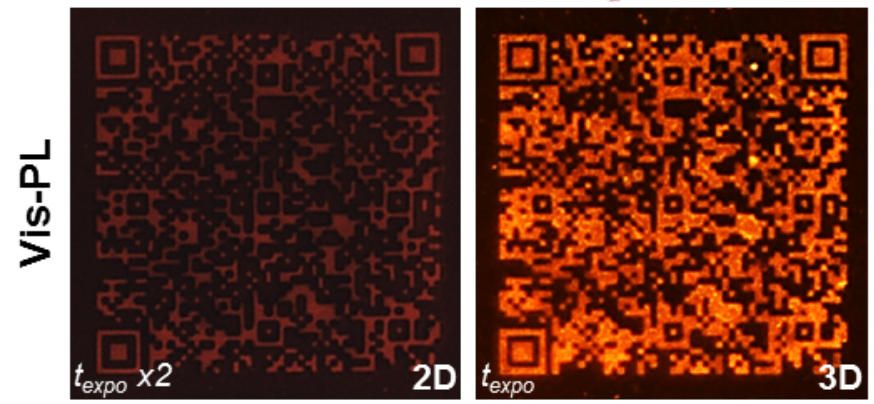

(b)

SEM

(c)

$20 \overline{\mu m}$
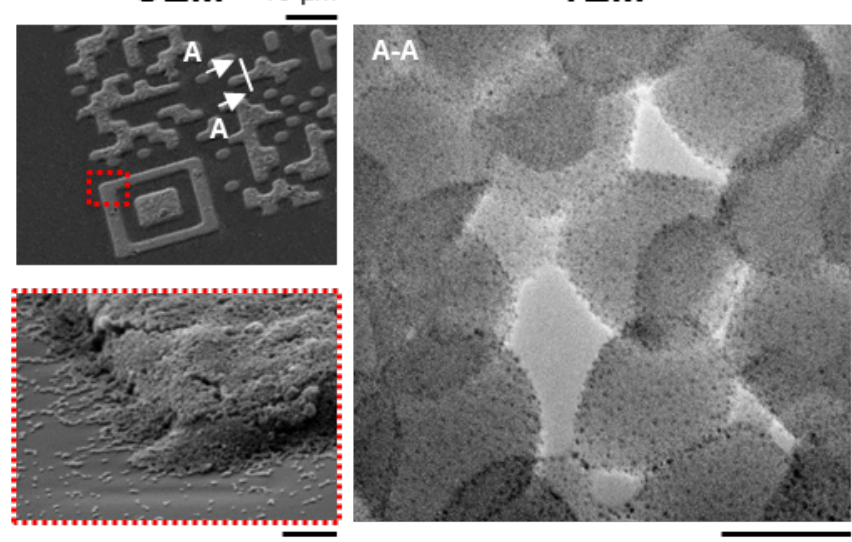

$1 \mu \mathrm{m}$

$50 \mathrm{~nm}$

Figure 4. (a) Anti-counterfeiting QR codes composed of 185 alphanumeric characters and constructed from (left) a 2D directed assembly of InP@ZnS NPs and (right) a 3D directed assembly of $\mathrm{SiO}_{2}-\operatorname{InP} @ \mathrm{ZnS} \mathrm{HNPs}$ by e- $\mu \mathrm{CP}$ nanoxerography. They both emit at $631 \mathrm{~nm}$ under a blue $(460 \mathrm{~nm})$ LED irradiation. Pictures are taken at different exposure times $\left(t_{\text {expo }}\right)$.

(b) Tilted scanning electron microscopy (SEM) images of the HNP assembly case and (c) corresponding transmission electron microscopy (TEM) image observable through a cross-section of the 3D HNP assembly realized by focused ion beam (FIB). 


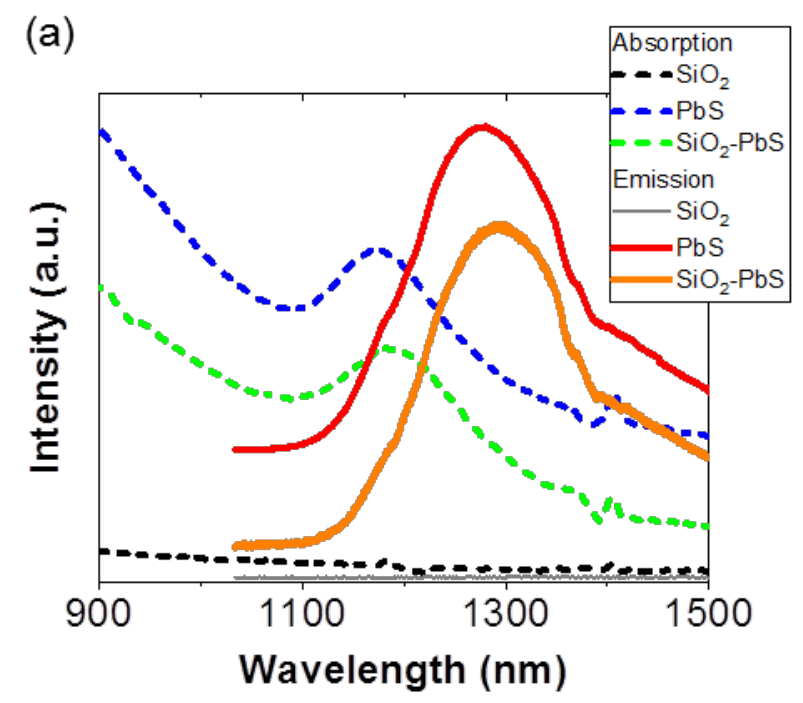

(b) PbS NPs $\mathrm{SiO}_{2}-\mathrm{PbS} \mathrm{HNPS}$
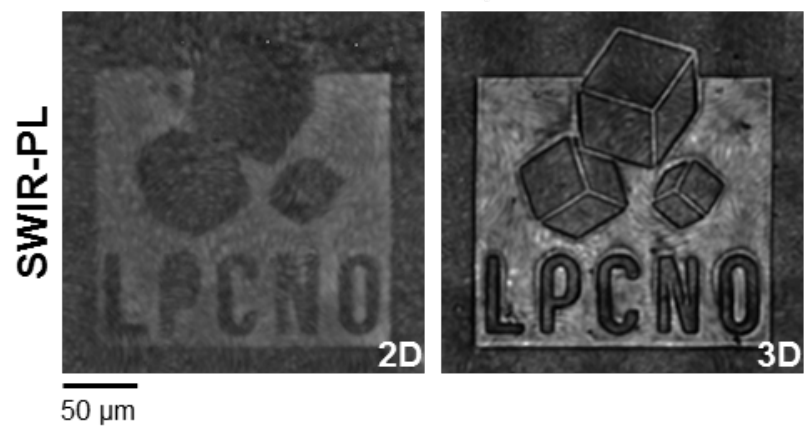

Figure 5. (a) Emission and absorption spectra of $\mathrm{SiO}_{2}, \mathrm{PbS} N \mathrm{NPs}$ and $\mathrm{SiO}_{2}-\mathrm{PbS}$ HNPs. (b) Anti-counterfeiting markers in the shape of the LPCNO logo constructed from (left) $2 \mathrm{D}$ assembly of $\mathrm{PbS} \mathrm{NPs}$ and (right) $3 \mathrm{D}$ assembly of $\mathrm{SiO}_{2}-\mathrm{PbS}$ HNPs. Both markers emit at $1286 \mathrm{~nm}$ under an infrared $(980 \mathrm{~nm})$ laser irradiation. SWIR-PL images are taken at the same exposure time.

\section{ASSOCIATED CONTENT}

Supporting Information. 


\section{AUTHOR INFORMATION}

\section{Corresponding Author}

*E-mail: epalleau@insa-toulouse.fr

\section{Author Contributions}

All authors contributed to the discussion and wrote the paper. All authors have given approval to the final version of the manuscript.

\section{Notes}

The authors declare no competing financial interests.

\section{Funding Sources}

This study has been supported through the grant NanoX $n^{\circ}$ ANR-17-EURE-0009 in the framework of the "Programme des Investissements d'Avenir" and by the French Ministry of the Armed Forces - Direction Générale de l'Armement.

\section{ACKNOWLEDGMENTS}

\section{REFERENCES}


[1] Y. Xia, P. Yang, Y. Sun, Y. Wu, B. Mayers, B. Gates, Y. Yin, F. Kim, H. Yan, OneDimensional Nanostructures: Synthesis, Characterization, and Applications, Advanced Materials. 15 (2003) 353-389. https://doi.org/10.1002/adma.200390087.

[2] A.N. Shipway, E. Katz, I. Willner, Nanoparticle arrays on surfaces for electronic, optical, and sensor applications, Chemphyschem. 1 (2000) 18-52. https://doi.org/10.1002/1439-7641(20000804)1:1<18::AID-CPHC18>3.0.CO;2-L.

[3] Y. Xia, B. Gates, Y. Yin, Y. Lu, Monodispersed Colloidal Spheres: Old Materials with New Applications, Advanced Materials. $12 \quad$ (2000) 693-713. https://doi.org/10.1002/(SICI)1521-4095(200005)12:10<693::AIDADMA693>3.0.CO;2-J.

[4] J. Zhu, M.C. Hersam, Assembly and Electronic Applications of Colloidal $\begin{array}{lllll}\text { Nanomaterials, } & \text { Advanced } & \text { Materials. } & 29 & \text { (2017) }\end{array}$ https://doi.org/10.1002/adma.201603895.

[5] J. Yang, M.K. Choi, D.-H. Kim, T. Hyeon, Designed Assembly and Integration of Colloidal Nanocrystals for Device Applications, Advanced Materials. 28 (2016) 11761207. https://doi.org/10.1002/adma.201502851.

[6] Y. Xin, J. Zhou, G. Lubineau, A highly stretchable strain-insensitive temperature sensor exploits the Seebeck effect in nanoparticle-based printed circuits, Journal of Materials Chemistry A. 7 (2019) 24493-24501. https://doi.org/10.1039/C9TA07591G.

[7] P. Traiwatcharanon, K. Timsorn, C. Wongchoosuk, Flexible room-temperature resistive humidity sensor based on silver nanoparticles, Mater. Res. Express. 4 (2017) 085038. https://doi.org/10.1088/2053-1591/aa85b6.

[8] S. Praveenkumar, S. Manikandan, D. Lingaraja, T. Sugapriya, A Review of Doped and Undoped ZnO Nanoparticles for Fabrication of Gas Sensor, Sensor Letters. 16 (2018) 889-900. https://doi.org/10.1166/s1.2018.4049.

[9] P.J. Rodríguez-Cantó, R. Abargues, H. Gordillo, I. Suárez, V. Chirvony, S. Albert, J. Martínez-Pastor, UV-patternable nanocomposite containing CdSe and $\mathrm{PbS}$ quantum dots as miniaturized luminescent chemo-sensors, RSC Adv. 5 (2015) 19874-19883. https://doi.org/10.1039/C4RA02812K.

[10] J. Wang, Nanomaterial-based electrochemical biosensors, Analyst. 130 (2005) 421-426. https://doi.org/10.1039/B414248A.

[11] P.D. Howes, R. Chandrawati, M.M. Stevens, Colloidal nanoparticles as advanced biological sensors, Science. 346 (2014). https://doi.org/10.1126/science.1247390.

[12] D. Guo, G. Xie, J. Luo, Mechanical properties of nanoparticles: basics and applications, J. Phys. D: Appl. Phys. 47 (2013) 013001. https://doi.org/10.1088/00223727/47/1/013001.

[13] M. Segev-Bar, H. Haick, Flexible Sensors Based on Nanoparticles, ACS Nano. 7 (2013) 8366-8378. https://doi.org/10.1021/nn402728g.

[14] D. Tsoukalas, From silicon to organic nanoparticle memory devices, Philosophical Transactions of the Royal Society A: Mathematical, Physical and Engineering Sciences. 367 (2009) 4169-4179. https://doi.org/10.1098/rsta.2008.0280.

[15] R.J.C. Brown, M.J.T. Milton, Nanostructures and nanostructured substrates for surface-enhanced Raman scattering (SERS), Journal of Raman Spectroscopy. 39 (2008) 1313-1326. https://doi.org/10.1002/jrs.2030.

[16] W. Ren, G. Lin, C. Clarke, J. Zhou, D. Jin, Optical Nanomaterials and Enabling Technologies for High-Security-Level Anticounterfeiting, Advanced Materials. 32 (2020) 1901430. https://doi.org/10.1002/adma.201901430.

[17] M. Grzelczak, J. Vermant, E.M. Furst, L.M. Liz-Marzán, Directed Self-Assembly of Nanoparticles, ACS Nano. 4 (2010) 3591-3605. https://doi.org/10.1021/nn100869j. 
[18] B. Bhushan, Springer Handbook of Nanotechnology, Springer Science \& Business Media, 2010.

[19] N. Vogel, M. Retsch, C.-A. Fustin, A. del Campo, U. Jonas, Advances in Colloidal Assembly: The Design of Structure and Hierarchy in Two and Three Dimensions, Chem. Rev. 115 (2015) 6265-6311. https://doi.org/10.1021/cr400081d.

[20] E. Palleau, L. Ressier, Combinatorial Particle Patterning by Nanoxerography, $\begin{array}{lllll}\text { ADVANCED } & \text { FUNCTIONAL } & \text { MATERIALS. } & 28 & \text { (2018). }\end{array}$ https://doi.org/10.1002/adfm.201801075.

[21] N.M. Sangeetha, P. Moutet, D. Lagarde, G. Sallen, B. Urbaszek, X. Marie, G. Viau, L. Ressier, 3D assembly of upconverting NaYF4 nanocrystals by AFM nanoxerography: creation of anti-counterfeiting microtags, Nanoscale. 5 (2013) 9587-9592. https://doi.org/10.1039/C3NR02734A.

[22] L. Teulon, E. Palleau, D. Morales, D. Poirot, L. Ressier, Interactive Nanogel Marking at the Microscale for Security and Traceability Applications, Advanced Materials Technologies. 3 (2018) 1700244. https://doi.org/10.1002/admt.201700244.

[23] D. Morales, L. Teulon, E. Palleau, T. Alnasser, L. Ressier, Single-Step Binary Electrostatic Directed Assembly of Active Nanogels for Smart Concentration-Dependent Encryption, $\quad$ Langmuir. $\quad 34 \quad$ (2018) 1557-1563. https://doi.org/10.1021/acs.langmuir.7b03519.

[24] D. Poirot, R. Platel, T. Alnasser, F. Guerin, E. Palleau, L. Ressier, SmartphoneIdentifiable Photoluminescent Nanoparticle-Based Multilevel Secured Tags by Electrical Microcontact Printing, ACS Appl. Nano Mater. 1 (2018) 5936-5943. https://doi.org/10.1021/acsanm.8b01634.

[25] R. Diaz, E. Palleau, D. Poirot, N.M. Sangeetha, L. Ressier, High-throughput fabrication of anti-counterfeiting colloid-based photoluminescent microtags using electrical $\begin{array}{lllll}\text { nanoimprint lithography, } & \text { Nanotechnology. } & 25 & \text { (2014) } & 345302 .\end{array}$ https://doi.org/10.1088/0957-4484/25/34/345302.

[26] P. Moutet, P. Deram, N.M. Sangeetha, L. Ressier, Dynamics of Dielectrophoretic-ForceDirected Assembly of NaYF4 Colloidal Nanocrystals into Tunable Multilayered Micropatterns, J. Phys. Chem. Lett. 5 (2014) 2988-2993. https://doi.org/10.1021/jz501393v.

[27] E.A. Baquero, H. Virieux, R.A. Swain, A. Gillet, A. Cros-Gagneux, Y. Coppel, B. Chaudret, C. Nayral, F. Delpech, Synthesis of Oxide-Free InP Quantum Dots: Surface Control and H2-Assisted Growth, Chem. Mater. 29 (2017) 9623-9627. https://doi.org/10.1021/acs.chemmater.7b04069.

[28] M.D. Tessier, D. Dupont, K. De Nolf, J. De Roo, Z. Hens, Economic and Size-Tunable Synthesis of InP/ZnE (E = S, Se) Colloidal Quantum Dots., Chem. Mater. 27 (2015) 4893-4898. https://doi.org/10.1021/acs.chemmater.5b02138.

[29] I. Moreels, Y. Justo, B. De Geyter, K. Haustraete, J.C. Martins, Z. Hens, Size-Tunable, Bright, and Stable PbS Quantum Dots: A Surface Chemistry Study, ACS Nano. 5 (2011) 2004-2012. https://doi.org/10.1021/nn103050w.

[30] E. Palleau, N.M. Sangeetha, G. Viau, J.-D. Marty, L. Ressier, Coulomb Force Directed Single and Binary Assembly of Nanoparticles from Aqueous Dispersions by AFM Nanoxerography, ACS Nano. 5 (2011) 4228-4235. https://doi.org/10.1021/nn2011893.

[31] L. Ressier, V. Le Nader, Electrostatic Nanopatterning of PMMA by AFM Charge Writing for Directed Nano-Assembly, Nanotechnology. $19 \quad$ (2008) 135301. https://doi.org/10.1088/0957-4484/19/13/135301.

[32] T.B. Jones, Electromechanics of particles, Cambridge Univ Pr, 1995.

[33] J. Schindelin, I. Arganda-Carreras, E. Frise, V. Kaynig, M. Longair, T. Pietzsch, S. Preibisch, C. Rueden, S. Saalfeld, B. Schmid, J.-Y. Tinevez, D.J. White, V. Hartenstein, 
K. Eliceiri, P. Tomancak, A. Cardona, Fiji: an open-source platform for biological-image analysis, Nature Methods. 9 (2012) 676-682. https://doi.org/10.1038/nmeth.2019.

[34] E. Palleau, N.M. Sangeetha, L. Ressier, Quantification of the Electrostatic Forces Involved in the Directed Assembly of Colloidal Nanoparticles by AFM Nanoxerography, Nanotechnology. 22 (2011) 325603. https://doi.org/10.1088/09574484/22/32/325603.

[35] S. Zhang, C.I. Pelligra, X. Feng, C.O. Osuji, Directed Assembly of Hybrid Nanomaterials and Nanocomposites, Advanced Materials. 30 (2018) 1705794. https://doi.org/10.1002/adma.201705794.

[36] R. Costi, A.E. Saunders, U. Banin, Colloidal Hybrid Nanostructures: A New Type of Functional Materials, Angewandte Chemie International Edition. 49 (2010) 4878-4897. https://doi.org/10.1002/anie.200906010.

[37] Y. Wu, J. Xu, X. Liu, Controlled patterning of upconversion nanocrystals through capillary force, Journal of Rare Earths. 38 (2020) 546-551. https://doi.org/10.1016/j.jre.2020.01.015.

[38] P. Kumar, S. Singh, B.K. Gupta, Future prospects of luminescent nanomaterial based security inks: from synthesis to anti-counterfeiting applications, Nanoscale. 8 (2016) 14297-14340. https://doi.org/10.1039/C5NR06965C.

[39] M. Ha, J.-H. Kim, M. You, Q. Li, C. Fan, J.-M. Nam, Multicomponent Plasmonic Nanoparticles: From Heterostructured Nanoparticles to Colloidal Composite Nanostructures, Chem. $\quad$ Rev. $119 \quad$ (2019) 12208-12278. https://doi.org/10.1021/acs.chemrev.9b00234. 


\section{Synthesis of Hybrid Colloidal Nanoparticles for a Generic Approach to 3D Electrostatic Directed Assembly: Application to Anti-Counterfeiting}

Romain Platel ${ }^{1}$, Louis Vaure ${ }^{1}$, Etienne Palleau ${ }^{1 *}$, Simon Raffy ${ }^{1}$, François Guerin ${ }^{1}$, Delphine Lagarde $^{1}$, Robin Cours ${ }^{2}$, Cécile Marcelot ${ }^{2}$, Bénédicte Warot-Fonrose ${ }^{2}$, Céline Nayral ${ }^{1}$, Fabien Delpech $^{1}$, Laurence Ressier ${ }^{1}$

1 - LPCNO, Université de Toulouse, CNRS, INSA, UPS, 135 avenue de Rangueil, 31077 Toulouse, France

2 - CEMES-CNRS, Université de Toulouse, 29 rue Jeanne Marvig, 31055 Toulouse, France

* Author to whom correspondence should be addressed: epalleau@insa-toulouse.fr / +33(0)5.61.55.96.72

1/ AFM characterizations of 2D mono or bilayered assemblies of single InP@ZnS and PbS QDs by AFM nanoxerography 
Figure S1 illustrates directed assembly by AFM nanoxerography in the case of single (a) InP@ZnS QDs and (b) PbS QDs on positively charged $10 \mu \mathrm{m}$ square patterns (with injection voltages from left to right equal to $+40 \mathrm{~V}+55 \mathrm{~V}$ and $+70 \mathrm{~V}$ ). It is demonstrated that in both cases it is not possible to assemble more than a bilayer of QDs. Furthermore, increasing the injection voltages of charged patterns allows increasing the QD density of the assembly : we observe that the first monolayer (colored in red on the Figure) of both InP@ZnS and PbS QDs is getting denser; then a second layer (colored in blue on the Figure) appears and gets denser too.

(a) - InP@ZnS NPs

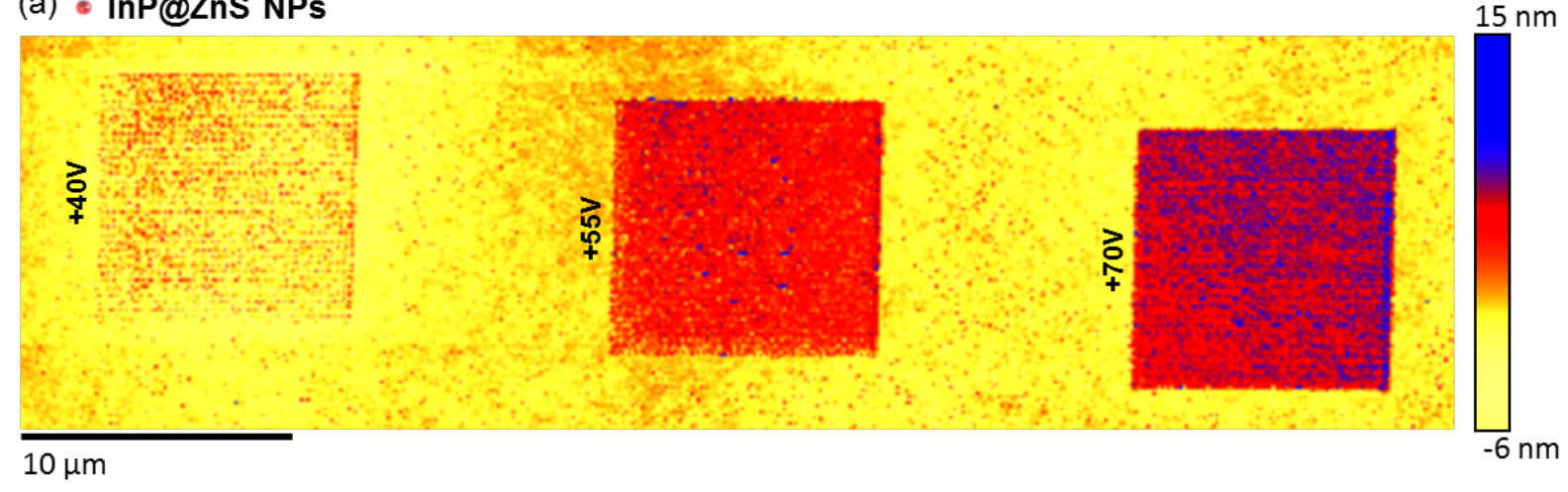

(b) PbS NPs

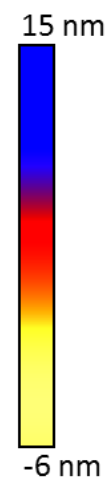

Figure S1. AFM characterizations of 2D mono or bilayered assemblies of single (a) InP@ZnS QDs and (b) PbS QDs by AFM nanoxerography.

\section{2/ Calculations on the numbers of emitters on 2D and 3D assemblies}


A/ Measured radii of InP@ $\mathrm{ZnS}$ and $\mathrm{SiO}_{2}$ NPs (based on height measurements from AFM images of assemblies of these NPs and on TEM images of these NPs in solution, as shown on Figure $\mathbf{2} \mathbf{b}$ of the manuscript):

$$
\begin{gathered}
R_{I n P @ Z n S}=1.9 \pm 0.2 \mathrm{~nm} \\
R_{\mathrm{SiO}_{2}}=24.4 \pm 2.4 \mathrm{~nm}
\end{gathered}
$$

Count of InP@ZnS NPs grafted on each $\mathrm{SiO}_{2}$ NP (based on TEM images of the hybrid $\mathrm{SiO}_{2}$-InP@ZnS NPs observable through cross-sections of $3 \mathrm{D}$ assemblies realized by focused ion beam (FIB), as shown on Figure $4 \mathbf{c}$ of the manuscript):

$$
N_{I n P @ Z n S / S i O_{2}}=150
$$

Maximum number of InP@ZnS NPs grafted on a single $\mathrm{SiO}_{2} \mathrm{NP}$ by considering a hexagonal closepacked grafting on the surface area of a sphere:

$$
N_{I n P @ Z n S / S i O_{2}(\max )}=\frac{d_{h c p(2 D)} * 4 \pi R_{\mathrm{SiO}_{2}}^{2}}{\pi R_{I n P @ Z n S}^{2}}=591
$$

With $d_{h c p(2 D)}=\frac{\pi}{2 \sqrt{3}} \cong 0.9069$ the highest average density (or packing factor) for a hexagonal closepacked layer of spheres in two dimensions (2D).

$\rightarrow$ If the maximum number of InP@ZnS NPs grafted on $\mathrm{SiO}_{2} \mathrm{NPs}$ would had been reached, the PL intensity of HNP assemblies would have been improved by an extra factor of 3.9 
B/ Theoretical maximum number of InP@ZnS NPs per 2D layer per square micrometer for a hexagonal close-packed (hcp) layer:

$$
N_{\text {InP@ZnS/layer }(\max )}=\frac{d_{h c p(2 D)}}{\pi R_{I n P @ Z n S}^{2}}=78718 N P s / \mu m^{2}
$$

Height of a layer of InP@ZnS NPs in a 3D assembly by considering a hexagonal close-packed structure (with $c$ the height of the unit hcp cell):

$$
h_{\text {layer }}=\frac{c}{2}=\sqrt{\frac{8}{3}} R_{\text {InP @ZnS }} \cong 3.1 \mathrm{~nm}
$$

Height of a 3D assembly composed of $N_{\text {layers }}$ of InP@ZnS NPs by considering a hexagonal closepacked structure:

$$
h_{\text {assembly }}=2 R_{\text {InP@ZnS }}+\left(N_{\text {layers }}-1\right) * h_{\text {layer }}
$$

Number of layers of InP@ZnS NPs in a 3D assembly:

$$
N_{\text {layers }}=\frac{h_{\text {assembly }}+h_{\text {layer }}-2 R_{\text {InP@ZnS }}}{h_{\text {layer }}}
$$

Maximum number of InP@ZnS NPs per square micrometer in a 3D assembly:

$$
\begin{gathered}
N_{I n P @ Z n S(\max )}=N_{\text {InP@ZnS/layer }(\max )} * N_{\text {layers }} \\
N_{\text {InP@ZnS (max })}=\frac{N_{\text {InP@ZnS/layer }(\max )}}{h_{\text {layer }}} *\left(h_{\text {assembly }}+h_{\text {layer }}-2 R_{\text {InP@ZnS }}\right)
\end{gathered}
$$

$\rightarrow$ The bilayered InP@ZnS NPs assembly, which has a PL intensity in red levels of 0.12 shown on Figure $3 \mathbf{d}$ of the manuscript (red diamond label), has a maximum thickness of $h_{\text {assembly }}=7.3 \mathrm{~nm}$ measured AFM, thus giving:

$$
N_{\text {InP@ZnS }(\max )}=167323 \mathrm{NPs} / \mu \mathrm{m}^{2}
$$


C/ Count of $\mathrm{SiO}_{2}$-InP@ZnS HNPs per layer per square micrometer (based on SEM and AFM images of assemblies of these hybrid NPs) for a close-packed layer:

$$
N_{S_{2} O_{2}-I n P @ Z n S / l a y e r}=270 N P S / \mu m^{2}
$$

Density of InP@ZnS NPs grafted on $\mathrm{SiO}_{2} \mathrm{NPs}$ :

$$
d_{I n P @ Z n S / S i O_{2}}=\frac{N_{I n P @ Z n S / S i O_{2}} * \pi R_{I n P @ Z n S}^{2}}{4 \pi R_{S i O_{2}}^{2}} \cong 23 \%
$$

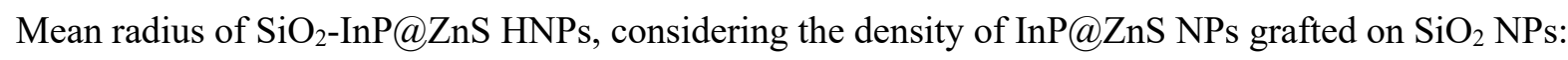

$$
R_{\mathrm{SiO}_{2}-\mathrm{InP} @ Z n S}=R_{\mathrm{SiO}_{2}}+d_{I n P @ Z n S / \mathrm{SiO}_{2}} * 2 R_{\text {InP@ZnS }} \cong 25.3 \mathrm{~nm}
$$

Height of a layer of $\mathrm{SiO}_{2}-\mathrm{InP} @ \mathrm{ZnS}$ HNPs in a 3D assembly by considering a hexagonal close-packed structure (with $c$ the height of the unit cell):

$$
h_{\text {layer }}=\frac{c}{2}=\sqrt{\frac{8}{3}} R_{\mathrm{SiO}_{2}-I n P @ Z n S} \cong 41.4 \mathrm{~nm}
$$

Number of $\mathrm{SiO}_{2}$-InP@ZnS HNPs per square micrometer in a 3D assembly:

$$
N_{\mathrm{SiO}_{2}-\mathrm{InP} @ Z n S}=N_{\mathrm{SiO}_{2}-\mathrm{InP} @ Z n S / \text { layer }} * N_{\text {layers }}
$$

Number of InP@ZnS NPs (grafted on $\mathrm{SiO}_{2} \mathrm{NPs}$ ) per square micrometer in a 3D assembly:

$$
N_{I n P @ Z n S}=N_{I n P @ Z n S / S i O_{2}} * N_{S i O_{2}-I n P @ Z n S}
$$


Height of a 3D assembly composed of $N_{\text {layers }}$ layers of $\mathrm{SiO}_{2}-\mathrm{InP} @ \mathrm{ZnS}$ NPs by considering a hexagonal close-packed structure:

$$
\begin{gathered}
h_{\text {assembly }}=2 R_{\mathrm{SiO}_{2}-\mathrm{InP} @ Z n S}+\left(N_{\text {layers }}-1\right) * h_{\text {layer }} \\
h_{\text {assembly }}=2 R_{\mathrm{SiO}_{2}-I n P @ Z n S}+\left(\frac{N_{\text {InP@ZnS }}}{N_{\text {InP@ZnS/SiO }} * N_{\text {SiO }}-\text { InP@ZnS/layer }}-1\right) * h_{\text {layer }}
\end{gathered}
$$

$\rightarrow$ For the equivalent number of NPs per square micrometer of the thickest bilayered InP@ZnS NPs assembly (as calculated above),

$$
h_{\text {assembly }} \cong 180 \mathrm{~nm}
$$

By considering this value and a linear fit of the data shown on Figure 3d of the manuscript, the corresponding PL intensity obtained in red levels is 0.14 .

This value is slightly higher than the measured one of 0.12 , probably because the number of InP@ZnS NPs of the thickest assembly is lower than the theoretical maximum number used for these calculations. 

layers

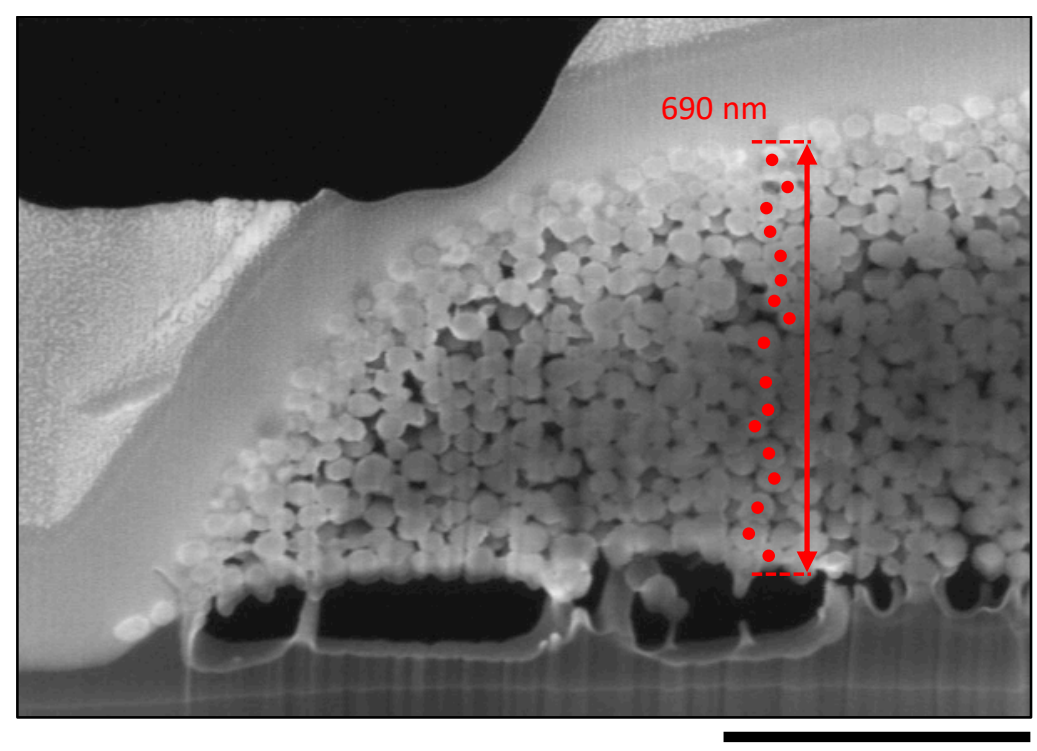

$500 \mathrm{~nm}$

Figure A. Scanning electron microscopy (SEM) image of a 3D $\mathrm{SiO}_{2}-\mathrm{InP} @ \mathrm{ZnS}$ NPs assembly crosssection realized by focused ion beam (FIB). A measured height of $690 \mathrm{~nm}$ is corresponding to a count of 17 layers.

With the formula used above, for $h_{\text {assembly }}=690 \mathrm{~nm}$, the following number of layers of $\mathrm{SiO}_{2}-$ InP@ZnS NPs in a 3D assembly is obtained:

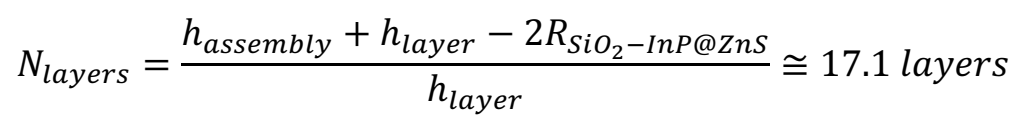

$\rightarrow$ The used formula is in good agreement with the measurements. 


\section{3/ Reading of a QR code obtained by nanoxerography of HNPs}

Figure S2 shows how the PL image of a QR code obtained by directed assembly of HNPs (from Figure 4a of the manuscript) could be easily read by a smartphone application. Some applications are powerful enough to directly read colored QR code (like i-nigma). If not, the raw photoluminescent signal recorded via the PL setup is first binarized using any image processing software (like Fiji [1]). Once converted to a black and white image, any code reader application would be able to decode the image and extract the information. Here it corresponds to the tracking information of a random product.

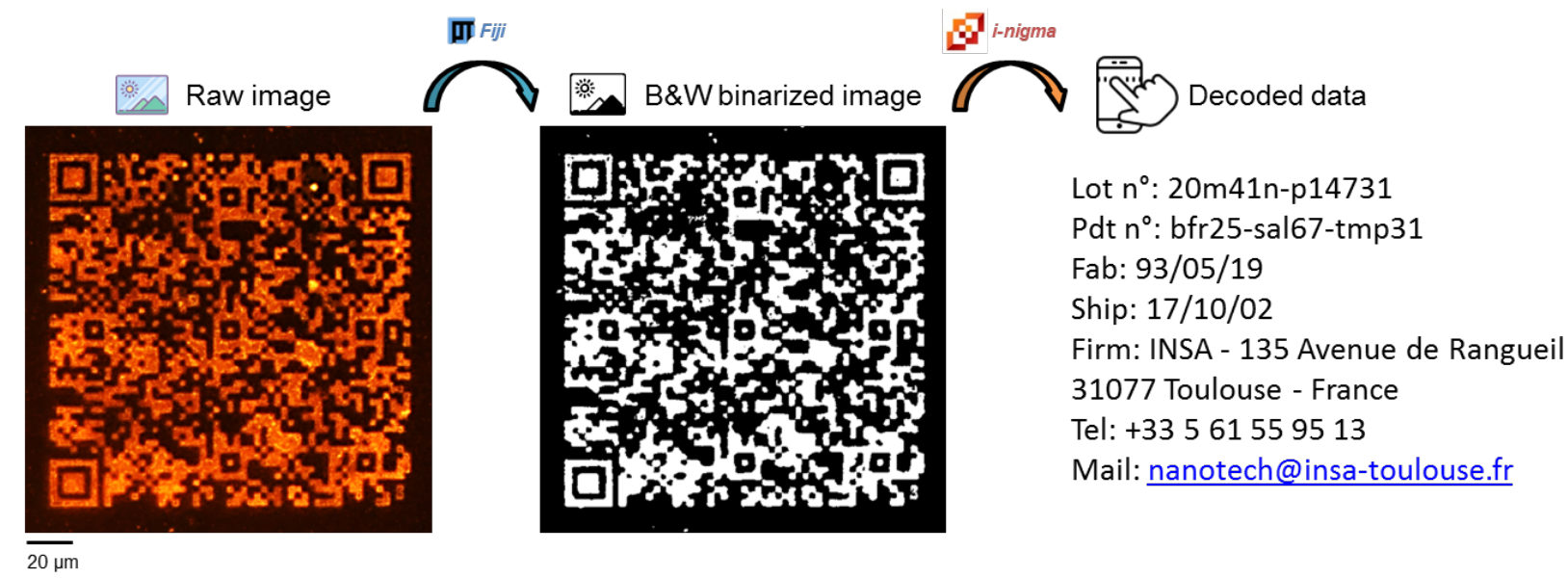

Figure S.2 Image processing and reading sequence of a QR code based on 3D assembly of HNPs.

[1] Schindelin, J.; Arganda-Carreras, I. \& Frise, E. et al. (2012), "Fiji: an open-source platform for biological-image analysis", Nature methods 9(7): 676-682, PMID 22743772, doi:10.1038/nmeth.2019 


\section{4/ Focused Ion Beam preparation of cross-section lamella of NP assemblies}

Figure S3 describes a typical protocol followed to obtain cross-section lamella of 3D NP assemblies.

(1) A 3D NP pattern of interest is selected.

(2) The assembly layer is protected by a $1.5 \mu \mathrm{m}$ thick Pt layer to avoid damages and charge accumulation during the thinning process.

(3) A cross-section of the whole stack is performed using a ThermoFisher Helios NanoLab 600i Focused Ion Beam (FIB) process. The final step is a low energy one to minimize irradiation damages and amorphization of the surfaces.

(4) The lamella is extracted using micromanipulator.

(5) It is then fixed on a support to be further thin down to $100 \mathrm{~nm}$ to get electron transparency.

(6) The lamella, further used for TEM observations, can be observed in the FEG-SEM included in the FIB system to probe the assembly from the bottom interface to the top surface.
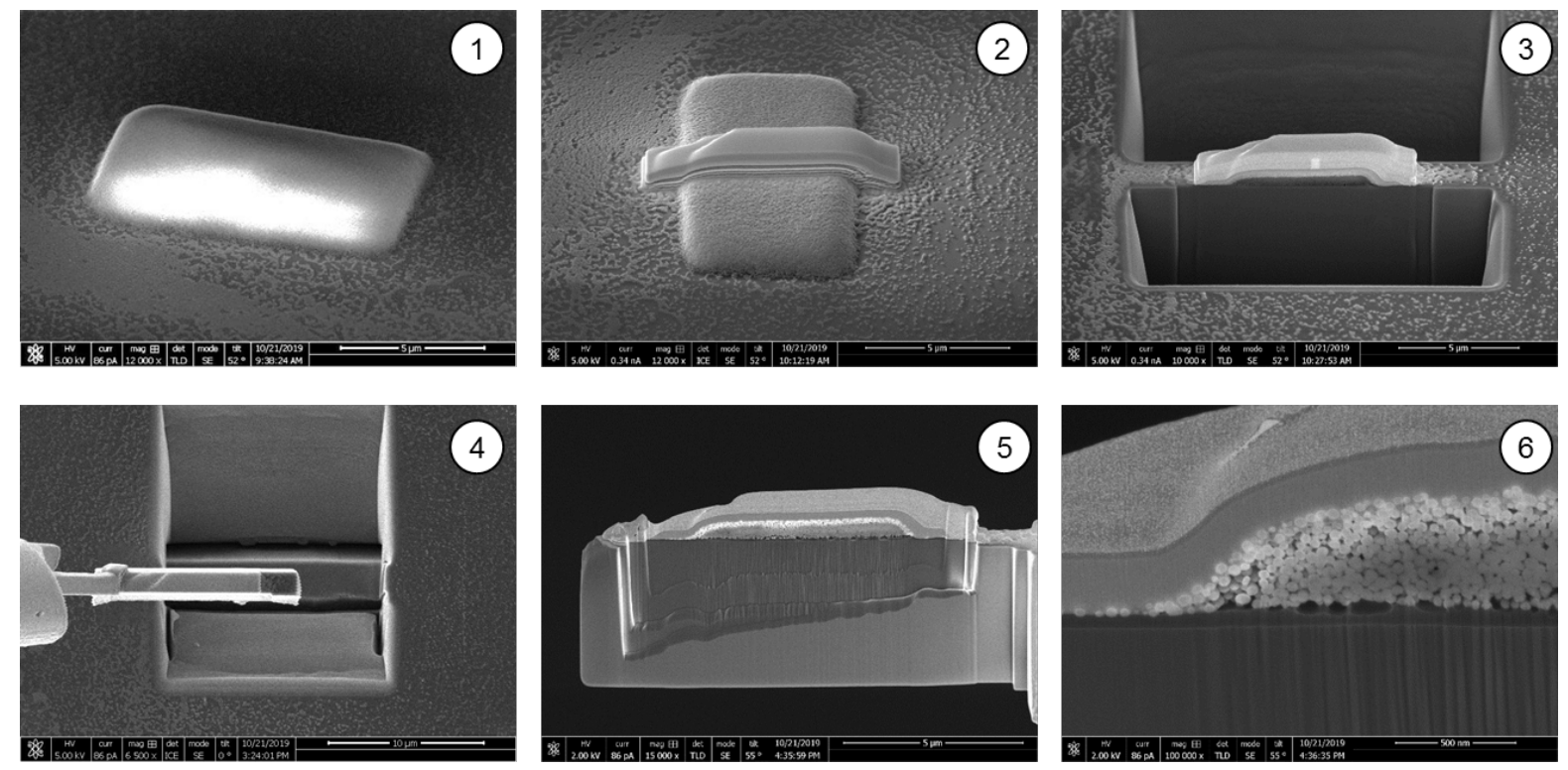

Figure S3. Focused Ion Beam preparation of cross-section lamella of 3D assemblies for further TEM characterization. 


\section{$5 /$ Influence of the surface potential of charge patterns and immersion time on the 3D assembly thickness}

Figure S4a illustrates the linear increase of the HNP assembly thickness with the increase of the surface potential of charge patterns. The surface potential of the charge patterns was carried out using Kelvin Force Microscopy (KFM) in lift" mode at a constant lift height of $30 \mathrm{~nm}$ with a fixed drive amplitude $\mathrm{V}_{\mathrm{AC}}$ of $500 \mathrm{mV}$. In our charge injection conditions, when the injection voltage amplitude varies from $+20 \mathrm{~V}$ to $+80 \mathrm{~V}$, the measured surface potential of the charges patterns typically ranges from $+200 \mathrm{mV}$ to $10 \mathrm{~V}$. These observations are in good agreement with previous works (cf. references [21] and [26]).

The assembly thickness as a function of the immersion time into the colloidal dispersion is shown in Figure S4b. Thickness measurements were carried out on various samples dipped for fixed immersion time into dispersions extracted from the same synthesis pot. They were performed by ex situ AFM topographical characterizations in tapping mode. Obtained assembly kinetics are also in good agreement with previously reported dynamics (cf. reference [26]). It is worth noting that the highest thickness value associated to the optimized immersion time value may differ a little from one HNP synthesis to another depending among other things on environmental conditions and small variations of dispersion concentrations. 

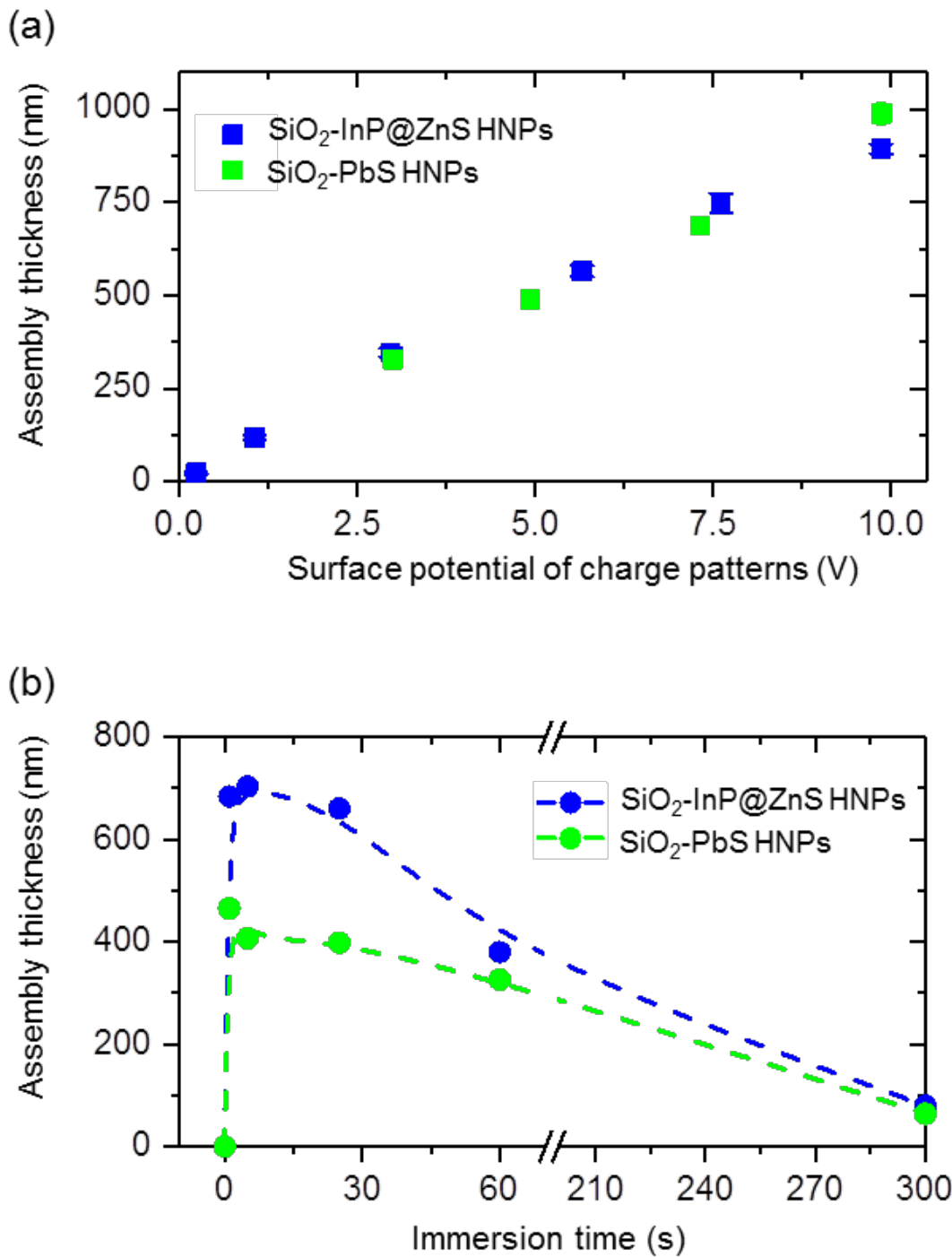

Figure S4. Influence of (a) the surface potential of charge pattern and (b) the immersion time into the colloidal solution on the assembly thickness in the case of $\mathrm{SiO}_{2}-\mathrm{InP} @ \mathrm{ZnS}$ and $\mathrm{SiO}_{2}-\mathrm{PbS} H N P s$ 


\section{6/ SEM and TEM characterizations of 3D assembly of $\mathrm{SiO}_{2}-\mathrm{PbS}$ hybrid nanoparticles}

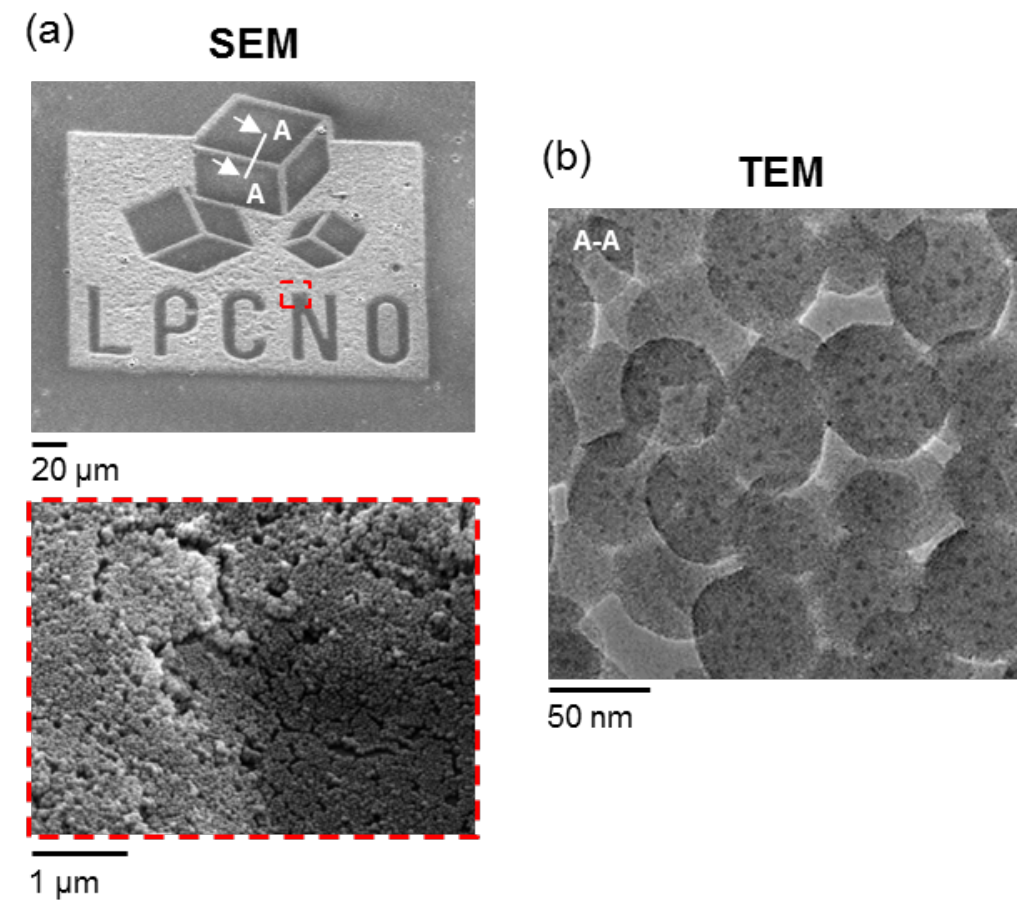

Figure S5. (a) Tilted low (top) and high (bottom) magnification scanning electron microscopy (SEM) images of a $3 \mathrm{D} \mathrm{SiO}_{2}$ - PbS HNP assembly in the shape of the LPCNO logo and (b) corresponding transmission electron microscopy (TEM) image observable through a cross-section realized by focused ion beam (FIB) of the assembly depicted in (a). 